\title{
Accelerometer Based Measurement of Body Movement for Communication, Play, and Creative Expression
}

\author{
Mark Nolan \\ Technological University Dublin, mark.nolan@tudublin.ie \\ Edward Burke \\ Technological University Dublin, ted.burke@tudubln.ie \\ Frank Duignan \\ Technological University Dublin, frank.duignan@tudublin.ie
}

Follow this and additional works at: https://arrow.tudublin.ie/teapotcon

Part of the Biomedical Devices and Instrumentation Commons

\section{Recommended Citation \\ Nolan, Mark, Burke, Edward, Duignan, Frank: Accelerometer Based Measurement of Body Movement for Communication, Play, and Creative Expression. eMBEC 2008, 4th European Conference of the International Federation for Medical and Biological Engineering ECIFMBE 2008 23-27 November 2008 Antwerp, Belgium.}

This Conference Paper is brought to you for free and open access by the tPOT: People Oriented Technology at ARROW@TU Dublin. It has been accepted for inclusion in Conference Papers by an authorized administrator of ARROW@TU Dublin. For more information, please contact arrow.admin@tudublin.ie, aisling.coyne@tudublin.ie,gerard.connolly@tudublin.ie. Funder: DIT Abbest

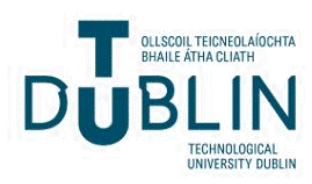




\title{
Accelerometer based measurement of body movement for communication, play, and creative expression.
}

\author{
M. Nolan ${ }^{1}$, E. Burke ${ }^{1}$ and F. Duignan ${ }^{1}$ \\ ${ }^{1}$ Dublin Institute of Technology, School of Electrical Engineering Systems, Dublin, Ireland
}

\begin{abstract}
This paper presents a newly designed wireless accelerometer-based movement measurement device. The device is capable of measuring activity ranging from gross body movements to more subtle vibrations emanating from the body, including laryngeal vibration and the mechanomyogram (mechanical vibrations from working muscles). The main body of the device, which is less than $20 \mathrm{~cm}^{3}$ in volume and weighs less than $50 \mathrm{~g}$, contains a microcontroller, wireless transceiver, battery, and one accelerometer. A supplementary accelerometer module is connected to the main device by thin wires. This module is very light weight and can therefore be directly attached to the skin to measure laryngeal vibration, mechanomyogram, or cardiac muscle movement.

The prototype device has been initially applied to facilitating play and creative expression by children with physical disabilities. For this purpose, the main module was adapted to be worn behind the ear while the supplementary accelerometer module is attached to the skin over the larynx.

In this paper, the device has been adapted to allow guidance of a radio controlled toy car. Direction is controlled by tilting the head, as measured by the accelerometer in the main device. The supplementary accelerometer is used to measure vocal pitch which controls the car's speed. The device has also been adapted to control a music synthesizer, with frequency of phonation controlling musical pitch while head position, which is measured by the main device's accelerometer, controls another parameter such as timbre.

Preliminary user trials with five subjects were carried out in these two applications and the results are presented. The system is also readily amenable to adaptation for other applications such as wheelchair navigation, mouse pointer control, or computer game input.
\end{abstract}

Keywords - Accelerometer, Wireless Sensors, Augmentative Communication, Man-Machine Interfaces, Universal Design.

\section{INTRODUCTION}

Play is a key part of a child's development, during which a child utilizes rules and their imagination to expand their conceptual ideas and acquire abstract thought [1]. Modern smart toys provide extra dimensions for a child's play and interaction with a toy through the use of motion, pressure and auditory sensors. Children with physical disabilities, who cannot interact with these toys, are at a distinct disadvantage. It has even been found that limiting a child's ability to interact with a toy, as would be the case with a child with a disability, can lead to regression [2]. Similarly, creative expression, through the medium of music provides several benefits for the development of children in areas such as language, reasoning and spatial intelligence [3]. Most children with physical disabilities are unable to play an instrument which has multiple operations and requires a player to have sufficient dexterity and coordination [4]. To remove this barrier for many children, it is therefore desirable to take a Universal Design [5] approach to facilitate accessibility with toys and musical instruments for children with physical disabilities.

\section{A. Universal Design}

The initiative of Universal Design facilitates the creation of products that are easily usable by the greatest range of people, regardless of their age or ability. There are seven principles of Universal Design and these are:
1. Equitable Use
2. Flexibility in Use
3. Simple and Intuitive Use
4. Perceptible Information
5. Tolerance for Error
6. Low Physical Effort
7. Size and Space for Approach and Use

With these principles in mind, a device was designed so that a child with a physical disability, would have the same level of control that any other user would have over a toy. If they were to compete against a peer, they could do so on a level playing field without stigmatizing or segregating them.

\section{B. Accelerometers}

In recent years, advances in sensor technology have created exciting opportunities in the area of man-machine interfaces. In particular, integrated circuit accelerometers have decreased in size and cost to the point where they are now routinely incorporated into portable consumer devices, including smartphones, game controllers, and personal digital assistants. In addition to the developments in ablebodied users' interaction with technology, these sensors provide powerful new means of interacting with technology for people with physical disabilities. 


\section{WIRELESS ACCELEROMETER DEVICE}

The device described in this paper consists of two modules. The main module contains a microcontroller, an accelerometer and a transceiver. The supplementary module is simply an accelerometer which is connected to the main device by thin wires. Both modules are powered by a $3 \mathrm{~V}$ battery which is contained in the main module. The device is capable of communicating wirelessly with a PC and also directly with a peripheral device, in this case, a radio controlled car. A block diagram for the system is shown in Fig. 1. The wireless protocol used is called Zigbee and is based on the IEEE 802.15.4 standard for wireless personal area networks [6]. Its advantages include low-cost, lowpower and secure networking.

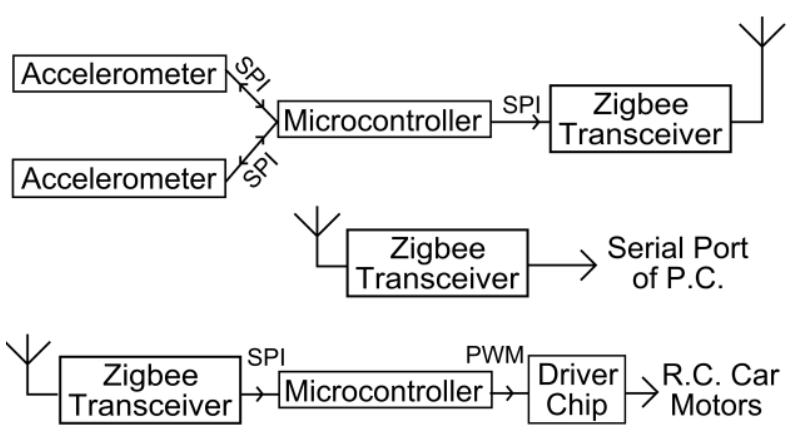

Fig. 1 Block Diagram of System

The microcontroller in the main module communicates with the accelerometers and the Zigbee transceiver via a Serial Peripheral Interface (SPI). It polls the accelerometers for the 16-bit values which represent the acceleration on each of the three axes. The axes are sampled at a frequency of $1280 \mathrm{~Hz}$. This satisfies the Nyquist-Shannon sampling theorem, as it is over twice the highest typical human vocal frequency which roughly ranges from $80 \mathrm{~Hz}$ to $1100 \mathrm{~Hz}$ [7].

The Zigbee network processor is configured as an end device. Once it is wirelessly connected to a Zigbee network, the data is transmitted to the coordinator where it can be processed. This Zigbee network is operating in the unlicensed $2.4 \mathrm{GHz}$ range and gives an over-the-air raw data transmission rate of $250 \mathrm{~kb} / \mathrm{s}$. Taking into consideration packet acknowledgment time and various transmission overheads, the maximum useable data through-put is $\mathbf{1 1 5 . 5}$ $\mathrm{kb} / \mathrm{s}$ [8]. The in-door range of this wireless device is approximately $10 \mathrm{~m}$.

\section{A. Example Application 1: Radio Controlled Car}

Initially, the device has been adapted to allow guidance of a radio-controlled toy car where the car has been modified to act as a coordinator in the Zigbee network. Conventional radio-controlled cars have four controls; forward, backward, left and right. Direction, in this case, is controlled by tilting the head left or right, as measured by the accelerometer in the main device which is clipped on behind the ear. The supplementary accelerometer is used to measure the frequency of phonation and is attached to the skin over the trachea and below the Cricoid cartilage as shown in Fig. 2. This frequency of phonation is calculated by a microcontroller in the main module using a software pitch detector. The speed of the car, which is dependent on this frequency, is altered by a pulse width modulated signal from the microcontroller in the car. This signal is sent into a driver chip which powers the motors.

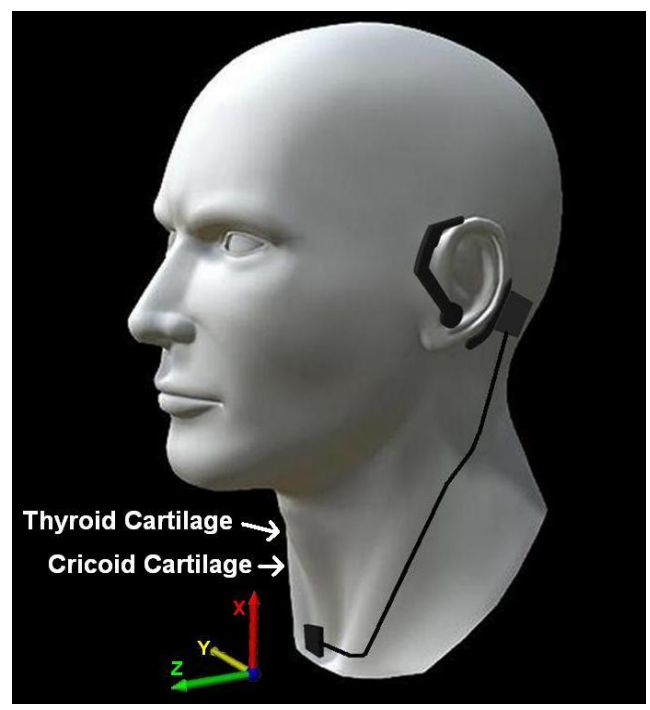

Fig. 2 Model of Device [9]

The transmitter in the toy car, which is typical of standard toy cars, can ideally transmit approximately twelve packets of data per second [10]. Given that the wireless accelerometer device can send up $14 \mathrm{kB}$ of data per second, this is more than adequate to be able to control a radio controlled car. The mean reaction time of a human for light stimuli is $190 \mathrm{~ms}$ [11]. This would mean that the average user could only react up to 5.3 times a second.

\section{B. Example Application 1: Testing}

Able-bodied subjects were asked to perform a predefined task with a radio-controlled car by following a course as shown in Fig. 3. This course requires the user to use each of the seven navigational states in the car. These are stop, forward, reverse, forward-left, forward-right, reverse-left, and reverse-right. During the odd laps, the subject must steer the car into the turn marked with the 
number one, reverse into turn two and then continue on. On the even laps this is reversed so that the subject turns into turn two, reverse into turn one and then continues on. This demonstrates the reverse-left and reverse-right states.

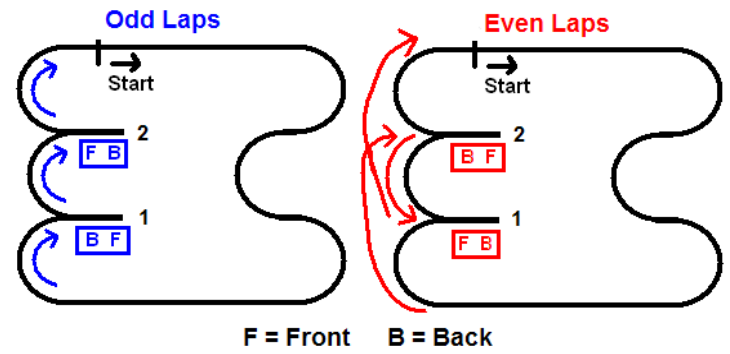

Fig. 3 Course

Each subject performed the task six times using the wireless accelerometer device (Task A). For comparative reasons, each subject was then asked to perform the same task using the conventional four button controller (Task B) on a different, but physically identical, car. The times taken for each lap are tabulated in Table 1 and the mean times per lap are plotted in Fig. 4.

Table 1 Results (in seconds)

\begin{tabular}{|r|c|c|c|c|c|c|c|c|c|c|c|c|}
\hline Lap & \multicolumn{2}{|c|}{$\mathbf{1}$} & \multicolumn{2}{|c|}{$\mathbf{2}$} & \multicolumn{2}{|c|}{3} & \multicolumn{2}{|c|}{$\mathbf{4}$} & \multicolumn{2}{|c|}{$\mathbf{5}$} & \multicolumn{2}{|c|}{$\mathbf{6}$} \\
\hline Task & A & B & A & B & A & B & A & B & A & B & A & B \\
\hline $\begin{array}{c}\text { Subject } \\
\mathbf{1}\end{array}$ & 63.6 & 31.8 & 75.2 & 29.4 & 53.2 & 33.3 & 64.2 & 26.6 & 50.7 & 23.9 & 52.6 & 24.7 \\
\hline $\mathbf{2}$ & 51.3 & 22.8 & 61.5 & 26.8 & 45.3 & 22.5 & 50.6 & 27.9 & 41.6 & 27.2 & 40.9 & 25.7 \\
\hline $\mathbf{3}$ & 55.9 & 50.2 & 58.3 & 37.4 & 53.7 & 37.9 & 53.5 & 28.8 & 51.3 & 36.5 & 48.2 & 25.7 \\
\hline $\mathbf{4}$ & 60.3 & 52.5 & 72.8 & 49.7 & 54.9 & 29.8 & 62.9 & 64.2 & 55 & 37.9 & 60.3 & 41 \\
\hline $\mathbf{5}$ & 85 & 49.6 & 90.1 & 35.8 & 76.7 & 58.9 & 82.6 & 59.3 & 70.9 & 85.4 & 71.5 & 75.1 \\
\hline
\end{tabular}

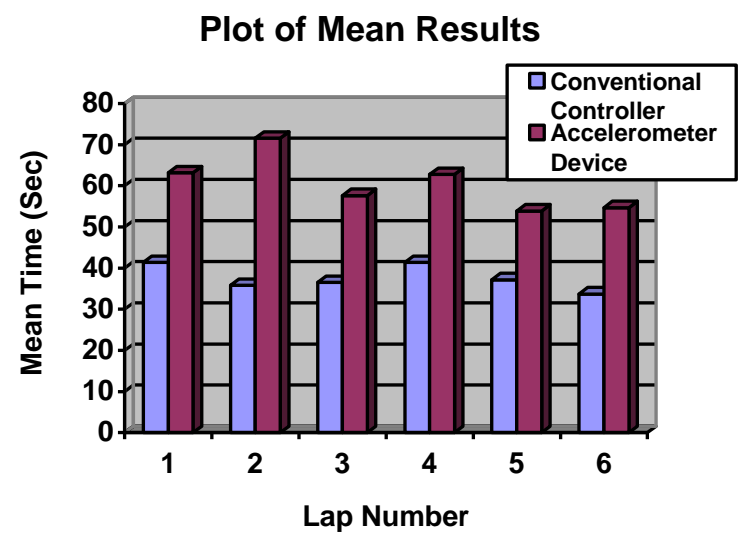

Fig. 4 Plot of Mean Results
It is evident from the bar graph in Fig.4 that although there is an initial time difference for the subjects to adapt to using the accelerometer device, over the course of the test, the times generally decrease. This is a sign of how intuitive the system is and, with more practice, a user could easily compete with a peer using a conventional controller.

\section{Example Application 2: Musical Synthesizer}

Using the same configuration, the device has also been adapted to control a music synthesizer. In this case a Zigbee coordinator is connected into the serial port of a computer. A midi synthesizer is initiated using the sound card in the computer. The data is read from the serial port buffer and the frequency of phonation and head position information is extracted from this. The frequency of phonation controls musical pitch while head position controls the instrument being used.

\section{Example Application 2: Testing}

To illustrate the ability of the accelerometer to record laryngeal vibrations, the experiment shown in Fig. 5 was devised. A sequence of three piano notes, C4, C5 and C6, which correspond to frequencies of $261.63 \mathrm{~Hz}, 523.25 \mathrm{~Hz}$ and $1046.5 \mathrm{~Hz}$, are played to a subject with an interval between them. The subject then attempts to sing each of the notes back during this interval, whilst both the laryngeal vibrations and the microphone input are recorded.

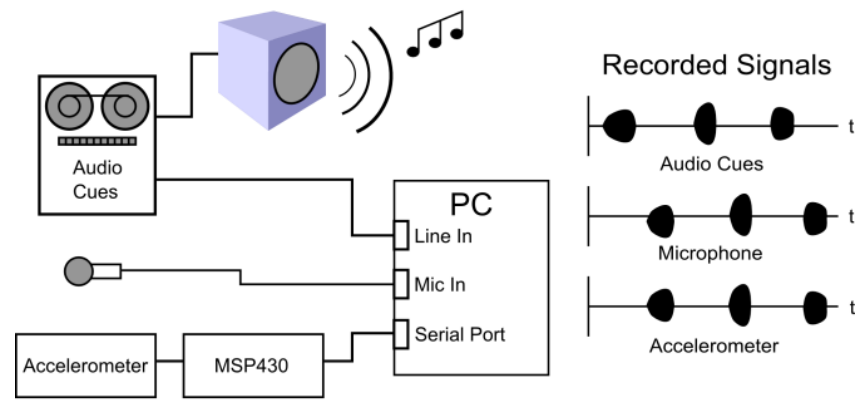

Fig. 5 Audio Block Diagram

The Fast Fourier Transform (FFT) of the three signals was calculated and the resulting normalized plots are shown in Fig. 6. It is evident from the FFT plots that there is good correlation between the microphone and accelerometer readings which validates the use of the accelerometer for pitch detection. It can also be noted, from the microphone recording, not only are harmonics of the human voice also recorded, but so is the background noise as well. 


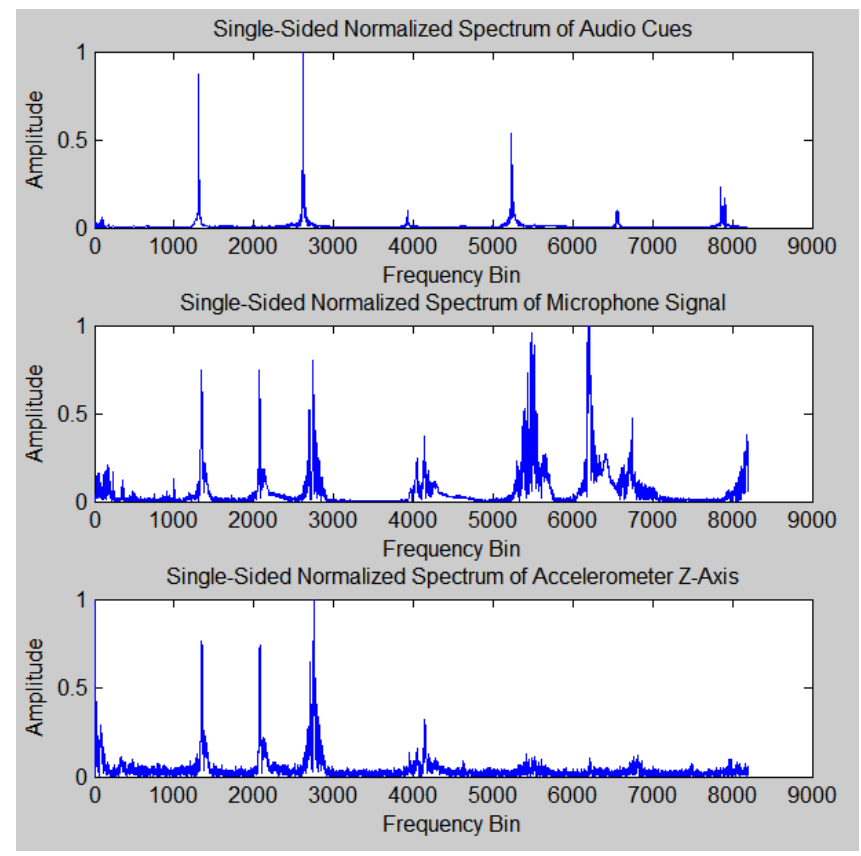

Fig. 6 Normalized FFT of the three signals

\section{ConClusions}

As shown in this paper, this device can assist children with physical disabilities to engage with toys and musicmaking activities that they would otherwise have limited access to. Unlike many traditional assistive technology user interface solutions [12], these sensors may facilitate design of software-definable user interfaces. This could allow for real Universal Design for play.

One of the advantages of using an accelerometer approach for control using laryngeal vibrations, compared to a microphone, is that the user has sole control over an instrument or device. Background noise is not recorded and therefore does not interfere with a user controlling an instrument or device. Another advantage is that the user wears a light weight and discrete device as opposed to a microphone which would need to be positioned adjacent to their mouth.

This system is readily amenable to adaptation for other user interface applications such as wheelchair navigation, mouse pointer control, or computer game input.

\section{AcKNOWLEDGMent}

M. Nolan would like to thank Dr. Edward Burke and Frank Duignan of the TeaPOT group in the Dublin Institute of Technology who provided the guidance and support for this project.

\section{REFERENCES}

1. Vygotskii, L S (1978) Mind in society : the development of higher psychological processes, Harvard University Press.

2. Barker R, Dembo T, Lewin K (1941) Frustration and Regression. An Experiment with Young Children. Univ. of Iowa Studies: Studies in Child Welfare, vol. XVIII, No. 1

3. Children's Music Workshop presents Music Education Online at http://www.childrensmusicworkshop.com/advocacy/12benefits.html

4. Craddock, G M, Knops, H (2003) Assistive Technology - Shaping the Future. IOS Press, pp 247-252

5. The Center for Universal Design, The Principles of Universal Design, North Carolina State University, 1997, at http://www.design.ncsu.edu/cud/pubs_p/docs/poster.pdf

6. Zigbee Alliance at http://www.zigbee.org/en/spec_download/

7. Titze, I R (1994) Principles of Voice Production. Prentice-Hall, Inc.

8. Burchfield, T R, Venkatesan, S, Weiner, D (2007) Maximizing Throughput in ZigBee Wireless Networks through Analysis, Simulations and Implementations. In Proceedings of the International Workshop on Localized Algorithms and Protocols for Wireless Sensor Networks Santa Fe, New Mexico, June 18-20, 2007. LOCALGOS 2007. CTI Press, Athens, pp 15-29

9. (Modified) http://www.digitaldrone.com/-sanchez/HighPolyHead.jpg

10. Toy car remote controller by Hangzhou Silan Microelectronics Co. at http://www.silan.com.cn/english/products/pdf/TX-2B(RX-2B)AY.pdf

11. Brebner, J T (1980) Reaction time in personality theory. In A. T. Welford (Ed.), Reaction Times. Academic Press, New York, pp. 309320

12. The Drake Music Project, Accessible Musical Devices (2005), at http://www.drakemusicproject.org/makepage. asp?page $=4 \mathrm{cAD}$

\begin{tabular}{|c|c|c|}
\hline & Author: & Mark Nolan \\
\hline & Institute: & Dublin Institute of Technology \\
\hline & Street: & Kevin Street \\
\hline & City: & Dublin 8 \\
\hline & Country: & Ireland \\
\hline & Email: & mark.nolan@dit.ie \\
\hline
\end{tabular}

